\title{
Dynamics and Conservation Laws of Generalized Chiral Solitons
}

\author{
Anjan Biswas ${ }^{*}$, , A.H. Kara ${ }^{2}$ and Essaid Zerrad ${ }^{3}$ \\ ${ }^{I}$ Department of Mathematical Sciences, Delaware State University, Dover, DE 19901-2277, USA \\ ${ }^{2}$ School of Mathematics and Centre for Differential Equations, Continuum Mechanics and Applications, University of \\ the Witwatersrand, Wits 2050, Johannesburg, South Africa \\ ${ }^{3}$ Department of Physics \& Pre-Engineering, Delaware State University, Dover, DE 19901-2277, USA
}

\begin{abstract}
This paper studies the generalized chiral solitons. The integration of the generalized version of the chiral nonlinear Schrödinger's equation is obtained. The conservation laws are also computed using the multiplier approach.
\end{abstract}

PACS Codes: 02.20.Sv; 02.30.Jr; 02.30.Ik.

Keywords: Integrability, conservation laws, Bohm potential.

\section{INTRODUCTION}

Chiral solitons appear in the study of Quantum Hall effect in Quantum Mechanics [1-12]. These are modeled by the chiral nonlinear Schrödinger's equation (cNLSE) [1-4, 12]. In fact a lot of studies have been going on in this area since the appearance of the Jackiw-Pie model [7, 8]. The dynamics of chiral solitons with Bohm potential has also been studied by the aid of He's semi-inverse variational principle [4]. The soliton perturbation theory was also studied with weak Bohm potential [1].

There are several mathematical techniques that have been developed in the past couple of decades to integrate the cNLSE and equations of those type which mathematically fall into the category of nonlinear evolution equtions. Some of these well known methods are Adomian decomposition method, exponential function method, Fan's $F$-expansion method, variational iteration method, semi-inverse variational principle and many more. These mathematical methods have truly been a blessing in this area of research. This paper will obtain the 1-soliton solution of the generalized version of the cNLSE by the ansatz method. Both topological soliton and non-topological soliton solutions will be obtained. Finally, the conservation laws will be derived for the generalized cNLSE.

\section{MATHEMATICAL ANALYSIS}

The generalized form of cNLSE that will be studied in this paper is given by

$i\left(q^{m}\right)_{t}+a\left(q^{m}\right)_{x x}+i b\left(q q_{x}^{*}-q^{*} q_{x}\right) q^{m}=0$

Here in (1) the dependent variable is $q$ while the independent variables are $x$ and $t$ which are the spatial and temporal variables respectively. Also, the parameter $m$

*Address correspondence to this author at the Department of Mathematical Sciences, Delaware State University, Dover, DE 19901-2277, USA; Tel: (302) 857-7913; Fax: (302) 857-7054; E-mail: biswas.anjan@gmail.com makes the cNLSE general. Here $m$ is a positive real number and the special case when $m=1$, equation (1) collapses back to the reagular cNLSE that was first proposed by Jackiw and Pie [7, 8]. The focus of this paper is to solve (1) by the ansatz method.

To start off, the following ansatz is proposed based on the traveling wave solution obtained by Wadati et al. for $m=1[11,12]$.

$q(x, t)=P(x, t) e^{i \phi(x, t)}$

where $P(x, t)$ represents the profile of the soliton while $\phi(x, t)$ is the phase component of the soliton that is defined as

$\phi(x, t)=-\kappa x+\omega t+\theta$

Here in (3), $\kappa$ represents the frequency of the soliton, while $\omega$ is the wave number of the soliton and finally $\theta$ is the phase constant. Thus, from (2),

$$
\begin{aligned}
& \left(q^{m}\right)_{t}=\left(m P^{m-1} \frac{\partial P}{\partial t}+i m \omega P^{m}\right) e^{i m \phi} \\
& \left(q^{m}\right)_{x x}=\left\{m P^{m-1} \frac{\partial^{2} P}{\partial x^{2}}-2 i m^{2} \kappa P^{m-1} \frac{\partial P}{\partial x}\right. \\
& \left.+m(m-1) P^{m-2}\left(\frac{\partial P}{\partial x}\right)^{2}-m^{2} \kappa^{2} P^{m}\right\} e^{i m \phi}
\end{aligned}
$$

and

$$
q q_{x}^{*}-q^{*} q_{x}=2 i \kappa P^{2}
$$

Substituting (2)-(6) into (1) and then decomposing into real and imaginary parts respectively yields

$$
\left(m \omega+a m^{2} \kappa^{2}\right) P^{m}+2 b \kappa P^{m+2}
$$


$-a m(m-1) P^{m-2}\left(\frac{\partial P}{\partial x}\right)^{2}-a m P^{m-1} \frac{\partial^{2} P}{\partial x^{2}}=0$

and

$\frac{\partial P}{\partial t}=2 a m \kappa \frac{\partial P}{\partial x}$

These equations (7) and (8) will be further analyzed in the following two subsections.

\subsection{Bright (Non-Topological) Solitons}

In this case, the hypothesis is [1-4]

$P(x, t)=A \operatorname{sech}^{p} \tau$

where

$\tau=B(x-v t)$

Here, in (9), $A$ is the amplidude of the soliton while $B$ is the inverse width of the soliton and $v$ is the velocity of the soliton. Therefore

$\frac{\partial P}{\partial t}=p v A B \operatorname{sech}^{p} \tau \tanh \tau$

$\frac{\partial P}{\partial x}=-p A B \operatorname{sech}^{p} \tau \tanh \tau$

$\frac{\partial^{2} P}{\partial x^{2}}=p^{2} A B^{2} \operatorname{sech}^{p} \tau-p(p+1) A B^{2} \operatorname{sech}^{p+2} \tau$

Thus, from (8), (11) and (12) it is possible to obtain

$v=-2 a m \kappa$

Also, equation (7), from (12) and (13), reduces to

$\left(m \omega+a m^{2} \kappa^{2}\right) A^{m} \operatorname{sech}^{m p} \tau+2 b \kappa A^{m+2} \operatorname{sech}^{(m+2) p} \tau$

$-\operatorname{am}(m-1) p^{2} A^{m} B^{2}\left(\operatorname{sech}^{m p} \tau-\operatorname{sech}^{m p+2} \tau\right)$

$-\operatorname{amA}^{m} B^{2}\left\{p^{2} \operatorname{sech}^{m p} \tau-p(p+1) \operatorname{sech}^{m p+2} \tau\right\}=0$

Now, from (15), equating the exponents $(m+2) p$ and $m p+2$ gives

$(m+2) p=m p+2$

so that

$p=1$

Again, from (15), setting the coefficients of the linearly independent functions $\operatorname{sech}^{m p+j} \tau$, for $j=0,2$ to zero gives

$\omega=\operatorname{am}\left(B^{2}-\kappa^{2}\right)$

and

$B=A \sqrt{-\frac{2 b \kappa}{a m(m+1)}}$

which is the wave number and the soliton amplitude-width relation. From (19), it is possible to conclude that the nontopological solitons to (1) will exist provided $a b \kappa<0$

Thus the bright (non-topological) 1-soliton solution to (1) is given by

$q(x, t)=A \operatorname{sech}[B(x-v t)] e^{i(-\kappa x+\omega t+\theta)}$

where the amplitude-width relation is given by (19) and the soliton wave number is given by (18) while the soliton velocity is related to its frequency as in (14). These relations poses a constraint that is given by (20).

\subsection{Dark (Topological) Solitons} $11]$

For topological solitons the hypothesis is given by $[2,3$,

$$
P(x, t)=A \tanh ^{p} \tau
$$

where $\tau$ is the same as deined in (10). However, in this case the parameters $A$ and $B$ are free parameters and $v$ is still the soliton velocity. Theefore, in this case

$\frac{\partial P}{\partial t}=p v A B\left(\tanh ^{p+1} \tau-\tanh ^{p-1} \tau\right)$

$\frac{\partial P}{\partial x}=p A B\left(\tanh ^{p-1} \tau-\tanh ^{p+1} \tau\right)$

$\frac{\partial^{2} P}{\partial x^{2}}=p(p-1) A B^{2} \tanh ^{p-2} \tau-2 p^{2} A B^{2} \tanh ^{p} \tau$

$+p(p+1) A B^{2} \tanh ^{p+2} \tau$

Therefore (7) reduces to

$\left(m \omega+a m^{2} \kappa^{2}\right) A^{m} \tanh ^{m p} \tau+2 b \kappa A^{m+2} \tanh ^{(m+2) p} \tau$

$-\operatorname{am}(m-1) p^{2} A^{m} B^{2}\left(\tanh ^{m p-2} \tau-2 \tanh ^{m p} \tau+\tanh ^{m p+2} \tau\right)$

$-\operatorname{amA}^{m} B^{2}\left\{\begin{array}{l}p(p-1) \tanh ^{m p-2} \tau-2 p^{2} \tanh ^{m p} \tau \\ +p(p+1) \tanh ^{m p+2} \tau\end{array}\right\}=0$

Similarly from (26) as in the previous subsection, the same value of $p$ as in (17) is obtained. Therefore, from (26), $\tanh ^{m p-2} \tau$ must have its coefficient to be zero. This forces

$m=1$

This shows that for the generalized cNLSE, the topological solitons will exist only for $m=1$ and no other value of $m$ can be permitted for the topological soliton solutions to be valid. This is a very important observation that is being made, for the first time in this paper.

Similarly, from the linearly independent function $\tanh ^{m p+j} \tau$ for $j=1,2$, setting their coefficients to zero yields

$\omega=-a\left(\kappa^{2}+2 B^{2}\right)$

and

$$
B=A \sqrt{\frac{b \kappa}{a}}
$$


which is the relation between the free parameters $A$ and $B$. This relation (29) also imposes the constraint

$$
a b \kappa>0
$$

which must be valid for the topological soliton solutions to exist. Finally, substituting (23) and (24) into (8) yields the velocity of the soliton as

$v=-2 a \kappa$

Hence, for the existence of topological solitons of the generalized cNLSE, equation (1) must be modified to

$i q_{t}+a q_{x x}+i b\left(q q_{x}^{*}-q^{*} q_{x}\right) q=0$

whose topological 1-soliton solution is given by

$q(x, t)=A \tanh [B(x-v t)] e^{i(-\kappa x+\omega t+\theta)}$

where in (33) the relation between the free parameters is given by (29), while the wave number is given by (28) and the velocity of the soliton is (31). The condition for the existence of topological soliton is seen in (30).

\section{CONSERVATION LAWS}

One of the most important aspects of NLEEs is the conservation laws. Without these conserved quantities (integrals of motion), the study of any NLEEs is not complete. This is because the dynamics of the propagating waves, that are governed by these equations, is not completely understood without these laws.

In order to evaluate conserved quantities, we resort to the invariance and multiplier approach based on the well known result that the Euler-Lagrange operator annihilates a total divergence. Firstly, if $\left(T^{t}, T^{x}\right)$ is a conserved vector corresponding to a conservation law, then

$D_{t} T^{t}+D_{x} T^{x}=0$

along the solutions of the differential equation $(d e=0)$.

Moreover, if there exists a nontrivial differential function $Q$, called a 'multiplier', such that

$E_{q}[Q \cdot(d e)]=0$,

then

$Q \cdot(d e)=D_{t} T^{t}+D_{x} T^{x}$,

where $E_{q}$ is the Euler-Lagrange operator for some (conserved) vector $\left(T^{t}, T^{x}\right)$. Thus, a knowledge of each multiplier $Q$ leads to a conserved vector determined by, inter alia, a Homotopy operator [5, 9].

For a system $d e 1=0 \& d e 2=0, Q=(f, g)$, say, so that

$f .(d e 1)+g .(d e 2)=D_{t} T^{t}+D_{x} T^{x}$,

and

$E_{(u, v)}\left[D_{t} T^{t}+D_{x} T^{x}\right]=0$.

Here, either $T^{t}$ or $T^{t}$ is the conserved density.

\subsection{Conserved Densities}

To determine the conservation laws of the cNLSE, we split the equation into real and imaginary parts with $q=u+i v$ to get

$u_{t}+a v_{x x}-2 b v\left(v u_{x}-u v_{x}\right)=0$,

$-v_{t}+a u_{x x}-2 b u\left(v u_{x}-u v_{x}\right)=0$

so that the multipliers $(f, g)$ are determined by

$$
\begin{aligned}
& E_{(u, v)}\left[f \cdot\left(u_{t}+a v_{x x}-2 b v\left(v u_{x}-u v_{x}\right)\right)\right. \\
& \left.+g .\left(-v_{t}+a u_{x x}-2 b u\left(v u_{x}-u v_{x}\right)\right)\right]=0 .
\end{aligned}
$$

For our purposes, we determine these for $f$ and $g$ dependence on $\left(t, x, u, v, u_{x}, u_{x x}, u_{x x x}, v_{x}, v_{x x}, v_{x x x}\right)$. Following some tedious calculations for $(f, g)$, the conserved densities, thereafter, are determined to be $T^{t}$ (with the corresponding densities of the cNLSE $T^{t}$ ), see below.

(a). $(f, g)=(u,-v)$,

$$
\begin{aligned}
T^{t} & =\frac{1}{2}\left(u^{2}+v^{2}\right), \\
\Phi^{t} & =\frac{1}{2}|q|^{2} .
\end{aligned}
$$

(b). $(f, g)=\left(u_{x x},-v_{x x}\right)$,

$$
\begin{aligned}
& T^{t}=\frac{1}{2}\left(u u_{x x}+v v_{x x}\right), \\
& \Phi^{t}=\frac{1}{2}\left(q q_{x x}^{*}+q^{*} q_{x x}\right) .
\end{aligned}
$$

(c). $(f, g)=\left(b u(v)^{2}+b(u)^{3}+a v_{x},-b v(u)^{2}-b(v)^{3}+a u_{x}\right)$,

$$
\begin{aligned}
& T^{t}=\frac{1}{4}\left(b u^{4}+2 b u^{2} v^{2}+b v^{4}-2 a v u_{x}+2 a u v_{x}\right), \\
& \Phi^{t}=\operatorname{ai}\left(q q_{x}^{*}-q^{*} q_{x}\right)+b|q|^{4} . \\
& (f, g)=\left(2 b x(u)^{3}+2 b x u(v)^{2}+2 a x v_{x}+4(a)^{2} t u_{x x}\right. \\
& \left.+a v,-2 b x(v)^{3}-2 b x v(u)^{2}+2 a x u_{x}-4(a)^{2} t v_{x x}+a u\right) \\
& T^{t}=\frac{1}{2}\left(\begin{array}{l}
b x u^{4}+2 b x u^{2} v^{2}+2 a u\left(x v_{x}+2 a t u_{x x}\right) \\
+v\left(b x v^{3}-2 a x u_{x}+4 a^{2} t v_{x x}\right)
\end{array}\right), \\
& \Phi^{t}=\frac{1}{2}\left[b x|q|^{4}+\operatorname{aix}\left(q q_{x}^{*}-q^{*} q_{x}\right)+2 a^{2} t\left(q q_{x x}^{*}+q^{*} q_{x x}\right)\right] .
\end{aligned}
$$$$
\text { (d). }
$$

\subsection{Conserved Quanities}

From the conserved densities that are derived in the previous subsection, the conserved quantities are given as follows:

$$
\begin{aligned}
& I_{1}=\int_{-\infty}^{\infty} \Phi^{t} d x=\frac{1}{2} \int_{-\infty}^{\infty}|q|^{2} d x=\frac{A^{2}}{B} \\
& I_{2}=\int_{-\infty}^{\infty} \Phi^{t} d x=\frac{1}{2} \int_{-\infty}^{\infty}\left(q^{*} q_{x x}+q q_{x x}^{*}\right) d x=\frac{2 A^{2}}{3 B}\left\{2 B-3\left(\kappa^{2}+B^{2}\right)\right\}
\end{aligned}
$$




$$
\begin{aligned}
& I_{3}=\int_{-\infty}^{\infty} \Phi^{t} d x=\int_{-\infty}^{\infty}\left\{i a\left(q q_{x}^{*}-q^{*} q_{x}\right)+b|q|^{4}\right\} d x=\frac{4 A^{2}}{3 B}\left(b A^{2}-3 a \kappa\right) \\
& I_{4}=\int_{-\infty}^{\infty} \Phi^{t} d x=\int_{-\infty}^{\infty}\left\{\frac{b}{2} x|q|^{4}+\frac{i a}{2} x\left(q q_{x}^{*}-q^{*} q_{x}\right)+a^{2} t\left(q^{*} q_{x x}+q q_{x x}^{*}\right)\right\} \\
& =\frac{a^{2} t A^{2}}{3 B}\left\{2 B-3\left(\kappa^{2}+B^{2}\right)\right\}
\end{aligned}
$$

\section{CONCLUSIONS}

This paper integrates the generalized cNLSE by the soliton ansatz method. Both topological as well as nontopological 1-soliton solutions have been obtained. It has also been proved that for the existence of topological solitons, the generalized CLSE must reduce to the regular cNLSE. Finally, the multiplier method approach is used to calculate a few conserved quantities of the cNLSE.

These results lay the basic foundation for the generalized cNLSE. Later these results will be used to study the soliton perturbation theory with Bohm potential. Additionally, the generalized cNLSE with Bohm potential will be integrated by the aid of He's semi-inverse variational principle. Those results will be reported in future publications.

\section{ACKNOWLEDGEMENT}

The research work for the first author (AB) and the third author (EZ) was fully supported by the Army Research Office (ARO) along with the Air Force Office of Scientific
Research (AFOSR) under the award number: W54428-RTISP and these supports are genuinely and sincerely appreciated.

\section{REFERENCES}

[1] Biswas A. Perturbation of chiral solitons. Nclr Phys B 2009; 806: 457-61.

[2] Biswas A. Chiral solitons in $(1+2)$ dimensions. Int J Theo Phys 2009; 48: 3403-9.

[3] Biswas A. Chiral solitons with time-dependent coefficients. Int J Theo Phys 2010; 49: 79-83.

[4] Biswas A, Milovic D. Chiral solitons with Bohm potential by He's variational principle. Phys Atom Ncli 2011; 74: 781-3.

[5] Göktas U, Hereman W. Computation of conservation laws for nonlinear lattices. Phya D 1998; 123: 425-36.

[6] Griguolo L, Seminara D. Chiral solitons from dimensional reduction of Chern-Simons gauged nonlinear Schrödinger equation: classical and quantum aspects. Nucl Phys B 1998; 516, 467-98.

[7] Jackiw R, Pi S. Soliton solutions to the gauged nonlinear Schrödinger equation on the plane". Phys Rev Lett 1990; 64: 296972.

[8] Jackiw R, Pi S. Time-dependent Chern-Simons solitons and their quantization. Phys Rev D 1991; 44: 2524-32.

[9] Kara A. A symmetry invaraince analysis of the multipliers and conservation laws of the Jaulent-Miodek and families of systems of KdV-type equations. J Nonl Mthl Phys 2009; 16: 149-56.

[10] Lee J, Lin C, Pashev O. Shock waves, chiral solitons and semiclassical limit of one-dimensional anyons. Chaos, Solitons \& Fractals 2004; 19: 109-28.

[11] Nishino A, Umeno Y \& Wadati M. Chiral Nonlinear Schrödinger Equation. Chaos Soli Frct 1998; 9: 1063-69.

[12] Wadati M \& Sogo K. Gauge Transformations in Soliton Theory. J Phys Soc Jpn 1983; 52: 394-8. 